\title{
Electric-field-induced nuclear-spin flips mediated by enhanced spin-orbit coupling
}

\author{
Toshiki Sugimoto $^{1 \star}$ and Katsuyuki Fukutani ${ }^{1,2 \star}$
}

Molecules made of identical nuclei of non-zero spin exist in nuclear-spin modifications, and the interconversion of these spin isomers is often forbidden for isolated states ${ }^{1-3}$. The interconversion between the nuclear-spin modifications, however, is promoted by inhomogeneous magnetic fields, such as those present on the surfaces of magnetic materials ${ }^{4}$. Nuclear-spin conversion on diamagnetic and insulating solid substances, on the other hand, is generally considered improbable. Here we present the observation of nuclear-spin flips of $\mathrm{H}_{2}$ and $D_{2}$ occurring on amorphous solid water surfaces with time constants of $370_{-140}^{+340} \mathrm{~s}$ and $1,220_{-580}^{+2,980} \mathrm{~s}$, respectively. To explain these unexpected conversion processes, we propose a model of electric-field-induced nuclear-spin flips. In this model, giant and inhomogeneous electric fields present on the ice surface $^{5}$ mix the electronic states of opposite parities by the Stark effect ${ }^{6}$, and significantly enhance the spin-orbit couplings between the electronic singlet-triplet spin states of the molecules. By virtue of these effects, the intramolecular hyperfine contact interaction induces the nuclear-spin conversion. This concept should have implications for controlling nuclear magnetization using external electric fields ${ }^{7}$.

In molecules, the nuclear spins of identical nuclei are entangled with each other, thereby classifying the molecule into nuclearspin modifications according to the total nuclear $\operatorname{spin}^{1-3}(I)$. Interconversion between the modifications is typically so slow that different nuclear-spin states behave as independent molecules, which has attracted attention in molecular physics ${ }^{6}$ as well as in nuclear magnetism ${ }^{8}$. A straightforward way to accelerate the interconversion is to introduce a perturbation that mixes the nuclear-spin states. In the case of two spins, the perturbation represents an antisymmetric spin operator $\mathbf{i}_{a}-\mathbf{i}_{b}$ (Fig. 1), where $\mathbf{i}_{a}$ and $\mathbf{i}_{b}$ denote the nuclear spins of the two identical nuclei $a$ and $b$. Such a perturbation is realized by the inhomogeneous magnetic fields present on the surfaces of antiferromagnetic materials such as $\mathrm{FeO}\left(\mathrm{OH}\right.$ ) and $\mathrm{Cr}_{2} \mathrm{O}_{3}$ (ref. 4). Even without an apparent magnetic field on the surface, the hyperfine contact interaction between conduction electrons of metal surfaces and the molecular nuclear spins causes such a perturbation ${ }^{4,9}$. Although this catalytic mechanism is valid for diamagnetic metals such as $\mathrm{Cu}$ (ref. 10) and Ag (refs 11,12), it cannot be applied to diamagnetic and insulating solid substances composed of closed-shell atoms, molecules and ions. Thus, at present, there is no physical theory to describe nuclear-spin conversion on diamagnetic and insulating surfaces, and no direct experimental observations of such processes have been reported so far.

This has a crucial consequence in astrophysics: the dominant interstellar solid surface is amorphous solid water ${ }^{13}$ (ASW), a diamagnetic insulator. The relative population of the ortho $(I=1)$ and para $(I=0)$ nuclear-spin modifications of hydrogen ${ }^{1}\left(\mathrm{H}_{2}\right)$,

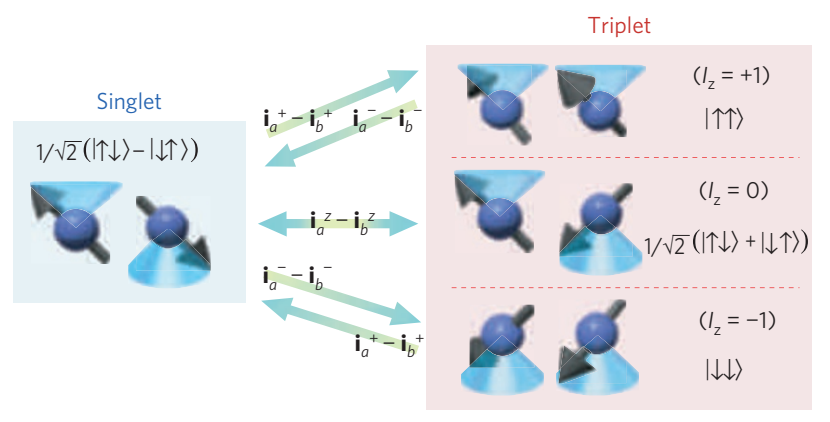

Figure 1 | Schematics of the singlet and triplet states of two identical spin $1 / 2$. The configurations of two proton spins are singlet for para- $\mathrm{H}_{2}(I=0)$ and triplet for ortho- $\mathrm{H}_{2}(I=1)$, respectively. $I_{z}$ is the $z$-component of the total nuclear spin. The wavefunction of the singlet state is antisymmetric and those of the triplet states are symmetric with respect to the permutation of the two identical nuclei (proton). The antisymmetric nuclear-spin operator $\mathbf{i}_{a}-\mathbf{i}_{b}$, where $\mathbf{i}_{a}$ and $\mathbf{i}_{b}$ are the nuclear-spin operators of proton $a$ and $b$, couples the singlet (para) state with the triplet (ortho) state. $i_{\mathrm{a}, \mathrm{b}}{ }^{ \pm}$represent creation and annihilation operators $i_{\mathrm{a}, \mathrm{b}}{ }^{x} \pm \sqrt{-1} i_{\mathrm{a}, \mathrm{b}}{ }^{y}$. In the case of two electron spins, the antisymmetric electron spin operator couples the electron-spin singlet state with the triplet states.

the Universe's most abundant molecule ${ }^{14}$, is thus expected to be preserved long after the formation of molecular clouds, that is the $\mathrm{H}_{2}$ nuclear-spin temperature carries important information on star formation ${ }^{15}$. As any nuclear-spin conversion of $\mathrm{H}_{2}$ on ASW surfaces would strongly affect the local astrophysical $\mathrm{H}_{2}$ nuclear-spin temperature, we have looked into the possibility of such interconversion processes in laboratory experiments.

Experimentally, we adopt resonance-enhanced multi-photon ionization $^{16}$ (REMPI) for the highly sensitive and rotational-stateselective detection of molecules in the gas phase. In the case of homonuclear diatomic molecules such as $\mathrm{H}_{2}$, the rotational quantum number $(J)$ is strongly coupled to the total nuclear-spin quantum number $(I)$; that is, the rotational levels of $\mathrm{H}_{2}$ with even and odd $J$ belong to the para $(I=0)$ and ortho $(I=1)$ modifications, respectively $^{1}$ (Fig. 2a). In combination with thermal desorption spectroscopy (TDS), the REMPI technique therefore enables us to measure the coverage evolution of physisorbed ortho- and para- $\mathrm{H}_{2}$ on surfaces (Supplementary Information Section SA). With this unique approach, we demonstrate quantitatively the nuclear-spin conversion of $\mathrm{H}_{2}$ and $\mathrm{D}_{2}$ isotopes on ASW surfaces and develop a new model for electric-field-induced nuclear-spin flips.

The experiments were carried out in an ultrahigh vacuum (UHV) chamber with a base operating pressure below $2 \times 10^{-8} \mathrm{~Pa}$. ASW films of 60 layers were grown on a $\mathrm{Ag}(111)$ single-crystal surface at $13 \mathrm{~K}$ by dosing $\mathrm{D}_{2} \mathrm{O}$ molecules. The ASW film, held at

${ }^{1}$ Institute of Industrial Science, The University of Tokyo, Komaba, Meguro-ku, Tokyo 153-8505, Japan, ${ }^{2}$ CREST, Japan Science and Technology Agency (JST), Komaba, Meguro-ku, Tokyo 153-8505, Japan. *e-mail: toshiki@iis.u-tokyo.ac.jp; fukutani@iis.u-tokyo.ac.jp. 

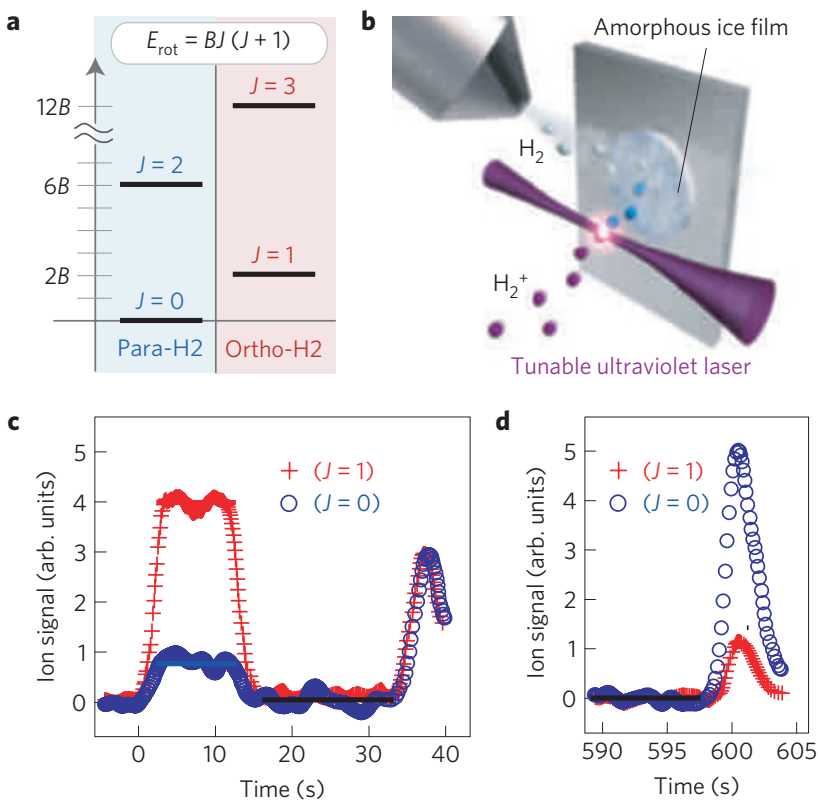

Figure 2 | Energy level diagram and measurement of ortho- and para- $\mathrm{H}_{2}$. a, Energy level diagram of the rotational motion of $\mathrm{H}_{2}$. The rotational energy is given by $B J(J+1)$, where $J$ is the rotational quantum number, and $B$ is the rotational constant of $\sim 7.5 \mathrm{meV}$ for $\mathrm{H}_{2}\left(\sim 3.8 \mathrm{meV}\right.$ for $\left.D_{2}\right)$ in the electronic ground state. Para- $\mathrm{H}_{2}$ (ortho- $\mathrm{D}_{2}$ ) has even $\mathrm{J}$, whereas ortho- $\mathrm{H}_{2}$ (para- $\mathrm{D}_{2}$ ) has odd $J$ (ref. 1). b. Schematic of the experimental set-up used for the dosage and J-state-selective thermal desorption spectroscopy of $\mathrm{H}_{2}$ on an amorphous ice surface. Tunable ultraviolet laser light for REMPI is focused by a spherical plano-convex glass lens $(f=400 \mathrm{~mm})$ in front of the sample at a distance of $\sim 2 \mathrm{~mm}$. $\mathbf{c}, \mathbf{d}, R E M P I$ data for $J=0$ and $J=1$ in a series of experiments from the initial $\mathrm{H}_{2}$ dosage to thermal desorption at $\sim 35 \mathrm{~s}$ (c) and at $\sim 600 \mathrm{~s}$ (d).

$10 \mathrm{~K}$, was exposed to a dosage $\left(6.5 \times 10^{15}\right.$ molecules $)$ of normal $\mathrm{H}_{2}$ at room temperature with an incidence angle of $45^{\circ}$ (Fig. 2b). The sample was heated to $30 \mathrm{~K}, 1-10 \mathrm{~min}$ after the dosage, at a heating rate of $3 \mathrm{~K} \mathrm{~s}^{-1}$ to induce thermal desorption of $\mathrm{H}_{2}$. During the dosage and the thermal desorption from the sample surface, $\mathrm{H}_{2}$ was $J$-state-selectively detected by REMPI (Fig. 2b). In the present experiment, only $J=0$ and $J=1$ desorption signals were detected because the ortho and para modifications are both at the lowest rotational states of $J=1$ and $J=0$ below $30 \mathrm{~K}$ (ref. 17). The laser set-up for REMPI is described in detail in a previous paper $^{11}$. Figure $2 \mathrm{c}$ shows typical REMPI data during a series of experiments. At $t=0 \mathrm{~s}, \mathrm{H}_{2}$ is introduced for $10 \mathrm{~s}$, which results in nearly constant signals for $J=1$ and $J=0$. The signals around $35 \mathrm{~s}$ correspond to the thermal desorption of $\mathrm{H}_{2}$ from the ASW surface. The desorption signal for $J=1$ is almost equal to that for $J=0$. The thermal desorption data taken at $600 \mathrm{~s}$ after the $\mathrm{H}_{2}$ dosage, on the other hand, show that the $J=1$ signal is substantially reduced whereas that for $J=0$ increases (Fig. 2d). These results indicate the ortho-para conversion of $\mathrm{H}_{2}$ on the amorphous ice surface at $10 \mathrm{~K}$. To evaluate the conversion time constant, we measured the time evolution of the $\mathrm{H}_{2}$ desorption intensities for $J=0$ and $J=1$ (Fig. 3a). As the residence time on the ASW surface increases, the intensity for $J=1$ decreases and that for $J=0$ increases. Also shown in the figure is the sum of the intensities for these two species, which is constant within the experimental accuracy. Fitting a single exponential function to the $J=1$ data gives a decay time constant of $370_{-140}^{+340} \mathrm{~s}$. It is worth noting that the ortho-para ratio just after the adsorption deviates from three, which is also observed on $\mathrm{Ag}$ surfaces ${ }^{11,12}$, and that the total number of $\mathrm{H}_{2}$ is preserved during the experiments even after the ortho-para
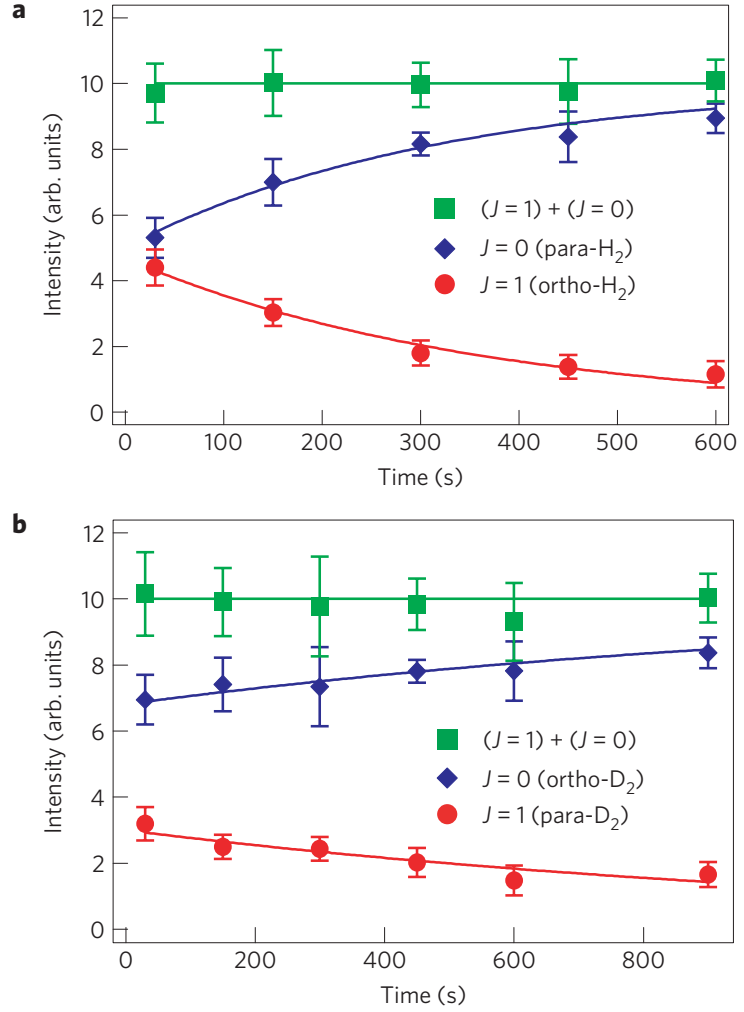

Figure 3 | Time evolution of the desorption intensities for $J=0$ and $J=1$ and their sum. a,b, $\mathrm{H}_{2}$ (a) and $\mathrm{D}_{2}$ (b) on the amorphous ice at $10 \mathrm{~K}$ (see Supplementary Section SC). The error bars represent the standard deviation of several independent measurements. The solid curves are fits using an exponential function, and the upper and lower limits of the time constant are evaluated by the chi-square test with a confidence level of 95\% (see text).

conversion, which is in contrast to the conversion of $\mathrm{H}_{2}$ on $\mathrm{Ag}$ surfaces $^{11,12}$. To clarify the isotope effect on the nuclear-spin flips on the ASW, we measure the time evolution of the desorption intensities for ortho- $\mathrm{D}_{2}(J=0: I=2,0)$ and para- $\mathrm{D}_{2}(J=1: I=1)$ (Fig. 3b). Fitting a single exponential function to the data gives a decay time constant of $1,220_{-580}^{+2,980} \mathrm{~s}$. These results indicate that the nuclear-spin flips of $\mathrm{H}_{2}$ and $\mathrm{D}_{2}$ do occur on ASW surfaces with the time constants given above.

Interconversion between the ortho and para modifications in the electronic ground $X^{1} \Sigma_{\mathrm{g}}{ }^{+}$state requires perturbations that induce the state mixing (Supplementary Information Section SB). On the basis that giant electric fields $\left(10^{10}-10^{11} \mathrm{~V} \mathrm{~m}^{-1}\right)$ with steep atomicscale gradients $\left(10^{20}-10^{21} \mathrm{~V} \mathrm{~m}^{-2}\right)$ are present on ASW surfaces ${ }^{5}$ and that $\mathrm{H}_{2}$ on ASW is infrared active through the induced electric dipole moment ${ }^{18}$, we propose that intramolecular Fermi contact coupling ${ }^{4}$ (IFCC; Supplementary Information Section SC) is effective for the nuclear-spin conversion through Stark coupling (SC) and spin-orbit coupling (SOC). Although these two couplings are normally small and can be neglected, we argue in the following that the SC is significant, and that the SOC is substantially enhanced in the molecules.

An external electric field mixes the electronic states with opposite parities (gerade-ungerade) and even-odd rotational states ${ }^{6}$. This is called SC, where the mixing is caused by the perturbation due to an electric-dipole moment. The typical value of the electric-dipole matrix element of $\mathrm{H}_{2}$ is $\sim e a_{0}$ (refs 19,20), where $e$ is the elementary charge and $a_{0}$ is the Bohr radius, respectively. At an electric field of $10^{10}-10^{11} \mathrm{~V} \mathrm{~m}^{-1}$, the energy scale of the Stark matrix element becomes of the order of an electronvolt, which is the same order 
as the energy difference between the electronic ground and excited states of $\mathrm{H}_{2}$. Under such a strong electric field, the SC coefficients become of the order of unity (Supplementary Information Section $\mathrm{SB})$. It should be noted that ionization of molecules can be neglected because the electric field is inhomogeneous on an atomic scale, which is in contrast to the homogeneous electric fields in intense light fields ${ }^{21}$.

The SOC is induced by the magnetic interaction of the electron orbital motion with its spin. The form of the intrinsic spin-orbit Hamiltonian of molecular hydrogen is $H_{\mathrm{SO}}{ }^{\text {int }}=\sum_{i=1,2} \xi_{i}\left[\boldsymbol{\ell}_{i} \cdot \mathbf{s}_{i}\right]$, where $\xi_{i}$ is the intrinsic spin-orbit interaction coefficient, and $\boldsymbol{\ell}_{i}$ and $\mathbf{s}_{i}$ are the angular momentum relative to the centre of mass of the molecule and the spin of the $i$ th electron, respectively ${ }^{22}$. Under an external electric field $\mathbf{E}$, an electron moving around the nuclei feels an additional spin-orbit interaction due to the centrifugal component of the electric field $\mathbf{E} \cdot \mathbf{r}_{i} /\left|\mathbf{r}_{i}\right|$. The form of the extrinsic spinorbit Hamiltonian is $H_{\mathrm{SO}}{ }^{\text {ext }}=\left(e \hbar^{2} / 2 m^{2} c^{2}\right) \sum_{i=1,2}\left(\mathbf{E} \cdot \mathbf{r}_{i} /\left|\mathbf{r}_{i}\right|^{2}\left[\boldsymbol{\ell}_{i} \cdot \mathbf{s}_{i}\right]\right.$ $\equiv \sum_{i=1,2} \eta_{i}(\mathbf{E})\left[\boldsymbol{\ell}_{i} \cdot \mathbf{s}_{i}\right]$, where $\mathbf{r}_{i}$ is the position vector of the $i$ th electron relative to the centre of mass, $m$ is the electron mass, $c$ is the light speed, and $\hbar$ is the Planck constant divided by $2 \pi$. We emphasize here that the expectation value of $\mathbf{E} \cdot \mathbf{r}_{i} /\left|\mathbf{r}_{i}\right|^{2}$ is non-zero only when $\mathbf{E}$ is inhomogeneous on an atomic scale. As a result, the spin-orbit interaction under an inhomogeneous electric field is expressed as $H_{\mathrm{SO}}{ }^{\text {tot }}=\sum_{i=1,2}\left\{\xi_{i}+\eta_{i}(\mathbf{E})\right\}\left[\boldsymbol{\ell}_{i} \cdot \mathbf{s}_{i}\right]$, where the first and second terms represent the intrinsic and extrinsic spin-orbit interactions, respectively. The first-order perturbation due to $H_{\mathrm{SO}}{ }^{\text {tot }}$ lifts the degeneracy of the electron spin triplet state according to the total angular momentum, whereas the secondorder perturbation combines the singlet and triplet states having the same total angular momentum, as schematically shown in Fig. $4 \mathrm{a}$. The SOC coefficient between the triplet $\left({ }^{3} \Pi_{1}\right)$ and singlet $\left({ }^{1} \Pi_{1}\right)$ states is described by $A / \Delta^{\mathrm{t}-\mathrm{s}}$, where $A=|\xi(n l)+\eta(\mathbf{E})|$ and $\Delta^{\mathrm{t}-\mathrm{s}}$ are the matrix element of $H_{\mathrm{SO}}{ }^{\text {tot }}$ and the energy difference between the triplet and the singlet states, respectively. The value of $\Delta^{\mathrm{t}-\mathrm{s}}$ for the $\left(1 s \sigma_{\mathrm{g}}\right)^{1}(n l \pi)^{1}$ electron configuration corresponding to single-electron excitation becomes smaller as $n$ or $l$ increases ${ }^{23-25}$, where $n$ and $l$ are the main and azimuthal quantum numbers in the united atom picture. This can be intuitively understood by considering that the electron wavefunction has an extended feature at larger $n$ and $l$ (ref. 23), and that the singlet-triplet energy difference reflects the electron-electron repulsion ${ }^{22}$. As the intrinsic SOC is determined by the electron-nucleus attractive Coulomb force, $\xi(n l)$ also decreases as $n$ or $l$ increases. On the other hand, the value of the extrinsic SOC is almost independent of $n$ or $l$, with $\eta(\mathrm{E})$ being $\sim 10^{-4} \mathrm{eV}$ in an electric field gradient of $10^{20}-10^{21} \mathrm{~V} \mathrm{~m}^{-2}$. Consequently, at high- $n, l$ states, the SOC coefficient of $A / \Delta^{\mathrm{t}-\mathrm{s}}$ leads to $\eta(\mathbf{E}) / \Delta^{\mathrm{t}-\mathrm{s}}$. This is an enhancement of the SOC induced by a giant and inhomogeneous electric field. In the case of the $\left(1 s \sigma_{\mathrm{g}}\right)^{1}\left(4 d \pi_{\mathrm{g}}\right)^{1}$ configuration, the SOC coefficient of $r^{3} \Pi_{\mathrm{g}}$ with $R^{1} \Pi_{\mathrm{g}}$ can be enhanced to the order of 0.1 as a result of the small energy difference $\Delta^{\mathrm{t}-\mathrm{s}} \sim 0.7 \mathrm{meV}$ (refs 24,25).

Figure $4 \mathrm{~b}$ shows a possible channel for effective mixing of the ortho- $\mathrm{H}_{2}(I=1: J=1)$ with the para- $\mathrm{H}_{2}(I=0: J=0)$ in the $X^{1} \Sigma^{+}{ }_{\mathrm{g}}$ state under giant electric fields $\left(10^{10}-10^{11} \mathrm{~V} \mathrm{~m}^{-1}\right)$ with steep atomic-scale gradients $\left(10^{20}-10^{21} \mathrm{~V} \mathrm{~m}^{-2}\right)$. The SC is as large as the order of unity, which leads to actual coupling coefficients of $1 / \sqrt{N}$, where $N$ denotes the number of the coupling states. With the IFCC and the enhanced SOC (ESOC) coefficients of $\sim 10^{-5}$ and $\sim 10^{-1}$ for the $B^{1} \sum_{\mathrm{u}}{ }^{+}-a^{3} \sum_{\mathrm{g}}{ }^{+}$and $r^{3} \Pi_{\mathrm{g}}-R^{1} \Pi_{\mathrm{g}}$, respectively, the total coupling coefficient can reasonably be estimated to be $\sim 10^{-8}$ for $\mathrm{H}_{2}$. The magnetic dipole moment of the deuteron is about three times smaller than that of the proton ${ }^{9}$, which results in a smaller total coupling coefficient for $\mathrm{D}_{2}$ than for $\mathrm{H}_{2}$ (Supplementary Information Section SC). As the deuteron nucleus has a quadrupole moment of $\sim 0.28 \mathrm{efm}^{2}$ (ref. 8), the nuclear quadrupole interaction with the electric field gradients leads to the $\mathrm{D}_{2}$ para-ortho coupling a

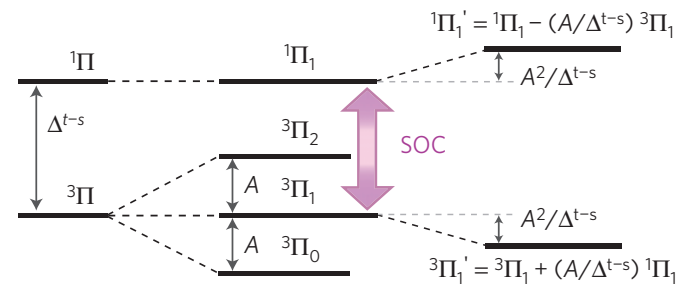

b

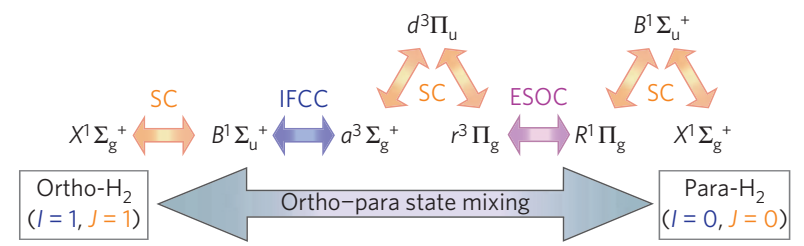

Figure 4 | SOC and the ortho-para state mixing under giant and inhomogeneous electric fields. a, An energy level diagram of the electron spin singlet and triplet states in a $\Pi$ state. See main text for details of the nomenclature. $\mathbf{b}$, An effective mixing channel of the ortho- $\mathrm{H}_{2}(I=1: J=1)$ with the para- $\mathrm{H}_{2}(I=0: J=0)$ in the electronic ground $X^{1} \Sigma_{\mathrm{g}}{ }^{+}$state via the electronically excited states through IFCC, SC and ESOC.

between $J=1$ and $J=0$ in the $X^{1} \Sigma_{\mathrm{g}}{ }^{+}$states. The nuclear quadrupole matrix element of $\sim 10^{-11} \mathrm{eV}$ and the energy difference of $\sim 7.5 \mathrm{meV}$ leads to the nuclear quadrupole coupling coefficient of $\sim 10^{-9}$, which is similar to the coupling due to the IFCC-SC-ESOC mechanism. From these coupling coefficients, $\left(\sim 10^{-8}\right.$ for $\mathrm{H}_{2}$ and $\sim 10^{-9}$ for $\mathrm{D}_{2}$ ), the order of the conversion time of $\mathrm{H}_{2}$ and $\mathrm{D}_{2}$ is estimated to be $\sim 10^{2}$ s and $\sim 10^{3}$ s (refs 4,9 ), respectively, which is in good agreement with our experimental results. Finally, it is worth noting that the magnetic interaction with water nuclear spins is not significant for conversion times of this magnitude (Supplementary Section SD).

The possibility of nuclear-spin conversion of $\mathrm{H}_{2}$ and $\mathrm{D}_{2}$ on diamagnetic and insulating ASW surfaces is demonstrated both experimentally and theoretically. Although the intrinsic SOC in $\mathrm{H}_{2}$ and $\mathrm{D}_{2}$ is small, giant electric fields with steep atomic-scale gradients significantly enhance the SOC and validate the IFCC without ionization of the molecules. In contrast to the conventional theory, our new model indicates that diamagnetic and insulating solid substances composed of closed-shell polar molecules and ions have the potential to serve as a catalyst for nuclear-spin conversion through their surface electric fields ${ }^{26}$, which also has profound implications for controlling nuclear magnetization by external electric fields. This new concept, at the present stage, is qualitative and further deep theoretical analysis will be a subject for future study. Furthermore, the spin-conversion time-constant obtained in this work will be a key parameter in understanding the dynamics of interstellar molecular clouds on the basis of the increasingly available data on the astronomical ortho/para ratio.

\section{Methods}

Preparation of the $\mathbf{A g ( 1 1 1 )}$ substrate. The $\mathrm{Ag}(111)$ single-crystal substrate was cleaned by cycles of $\mathrm{Ar}^{+}$bombardment at $500 \mathrm{eV}$ and annealing at $700 \mathrm{~K}$ for $1 \mathrm{~min}$ in a UHV environment. The surface cleanliness and crystalline order of the substrate were checked using Auger-electron spectroscopy and low-energy electron diffraction (LEED), respectively. Auger-electron spectra revealed that the substrate was chemically clean silver with no trace of oxygen contaminant to induce dissociative adsorption of water molecules. LEED revealed a clear $1 \times 1$ pattern with the three-fold symmetry of a face-centred cubic (111) surface structure. From these preparatory experiments, we confirmed that the substrate was chemically clean and well-ordered $\mathrm{Ag}(111)$. 
Preparation of the ASW film. The $\mathrm{D}_{2} \mathrm{O}$ water sample, with a purity of 99.96 atom $\% \mathrm{D}$ as purchased from ISOTEC, was carefully pre-degassed in a baked UHV gas line by repeated freeze-pump-thaw cycles. The ASW film of 60 bilayers was grown by $\mathrm{D}_{2} \mathrm{O}$ deposition through a molecular beam gas doser in the surface normal direction onto $\mathrm{Ag}(111)$ at a substrate temperature of $\sim 13 \mathrm{~K}$. The deposition rate was 0.11 bilayers per second. Here, the water coverage was normalized by the bilayer density $\left(\sim 1.1 \times 10^{15}\right.$ molecules $\left.\mathrm{cm}^{-2}\right)$ for the crystalline ice- $I_{\mathrm{h}}$. For the deposition rate used here, the ice film grown at $13 \mathrm{~K}$ is known to be amorphous $^{27,28}$. The ice film was annealed at $55 \mathrm{~K}$ to avoid any structural changes during the $\mathrm{H}_{2}$ adsorption and desorption experiments, which were conducted in the temperature range of $10-30 \mathrm{~K}$. The ice film was characterized by TDS and LEED. When the sample was heated from $13 \mathrm{~K}$ to $300 \mathrm{~K}$ with a heating rate of $2 \mathrm{~K} \mathrm{~s}^{-1}$, the zeroth-order single TDS peak was observed at around $170 \mathrm{~K}$, which was in good agreement with previous TDS studies of water on $\mathrm{Ag}(111)$ (ref. 29). Here, we emphasize that there was no desorption peak around $50 \mathrm{~K}$ corresponding to desorption of $\mathrm{O}_{2}$ (ref. 30). The LEED pattern of the ice film at $13 \mathrm{~K}$ revealed no diffraction spots, indicating that there was no ordered structure on the surface. From these preparatory experiments, we confirmed that the ice film was in an amorphous state.

Received 1 July 2010; accepted 10 November 2010; published online 30 January 2011

\section{References}

1. Farkas, A. Orthohydrogen, Parahydrogen and Heavy Hydrogen (Cambridge Univ. Press, 1935).

2. Pachucki, K. \& Komasa, J. Ortho-para transition in molecular hydrogen. Phys. Rev. A 77, 030501 (2008)

3. Hougen, J. T. \& Oka, T. Nuclear spin conversion in molecules. Science 310, 1913-1914 (2005)

4. Ilisca, E. Ortho-para conversion of hydrogen molecules physisorbed on surfaces. Prog. Surf. Sci. 41, 217-335 (1992).

5. Girardet, C. \& Toubin, C. Molecular atmospheric pollutant adsorption on ice: A theoretical survey. Surf. Sci. Rep. 44, 159-238 (2001).

6. Herzberg, G. Molecular Spectra and Molecular Structure I. Spectra of Diatomic Molecules (Krieger, 1950).

7. Eerenstein, W., Mathur, N. D. \& Scott, J. F. Multiferroic and magnetoelectric materials. Nature 442, 759-765 (2006).

8. Abragam, A. Principles of Nuclear Magnetism (Oxford Science, 1961).

9. Yucel, S. Theory of ortho-para conversion in hydrogen adsorbed on metal and paramagnetic surfaces at low temperatures. Phys. Rev. B 39, 3104-3114 (1989).

10. Svensson, K. \& Andersson, S. Fast ortho-para conversion of $\mathrm{H}_{2}$ adsorbed at copper surface step atoms. Phys. Rev. Lett. 98, 096105 (2007).

11. Fukutani, K. et al. Photostimulated desorption and ortho-para conversion of $\mathrm{H}_{2}$ on Ag surfaces. Phys. Rev. Lett. 90, 096103 (2003).

12. Niki, K., Ogura, S., Matsumoto, M., Okano, T. \& Fukutani, K. Effects of coadsorbed $\mathrm{O}_{2}$ on hydrogen ortho-para conversion on Ag surfaces. Phys. Rev. B 79, 085408 (2009).

13. Gibb, E. L., Whittet, D. C. B., Boogert, A. C. A. \& Tielens, A. G. G. M. Interstellar ice: The Infrared Space Observatory legacy. Astrophys. J. Suppl. Ser. 151, 35-73 (2004).

14. Habart, E. et al. Molecular hydrogen. Space Sci. Rev. 119, 71-91 (2005).

15. Neufeld, D. A. et al. Spitzer observations of HH 54 and HH 7-11: Mapping the $\mathrm{H}_{2}$ ortho-to-para ratio in shocked molecular gas. Astrophys. J. 649, 816-835 (2006).
16. Hou, W. M., Rinnen, K. D. \& Zare, R. N. Rotational and vibrational effects in the $E^{1} \Sigma_{\mathrm{g}}{ }^{+}-X^{1} \Sigma_{\mathrm{g}}{ }^{+}$two-photon transitions of $\mathrm{H}_{2}, \mathrm{HD}$ and $\mathrm{D}_{2}$. J. Chem. Phys. 95, 205-213 (1991).

17. Amiaud, L. et al. Measurement of the adsorption energy difference between ortho- and para- $\mathrm{D}_{2}$ on an amorphous ice surface. Phys. Rev. Lett. 100, 056101 (2008).

18. Hixson, H. G., Wojcik, M. J., Devlin, M. S., Devlin, J. P. \& Buch, V. Experimental and simulated vibrational spectra of $\mathrm{H}_{2}$ adsorbed in amorphous ice: Surface structures, energetics, and relaxations. J. Chem. Phys. 97, 753-767 (1992)

19. Spielfiedel, A. Ab initio calculation of electronic transition moments for singlet excited states of the $\mathrm{H}_{2}$ molecule. J. Mol. Spectrosc. 217, 162-172 (2003).

20. Staszewska, G. \& Wolniewicz, L. Transition moments among ${ }^{3} \Sigma$ and ${ }^{3} \Pi$ states of the $\mathrm{H}_{2}$ molecule. J. Mol. Spectrosc. 198, 416-420 (1999).

21. Akagi, A. et al. Laser tunnel ionization from multiple orbitals in $\mathrm{HCl}$. Science 325, 1364-1367 (2009).

22. Brion, H. L. \& Field, R. W. The Spectra and Dynamics of Diatomic Molecules (Academic, 2004)

23. Rothenberg, R. \& Davidson, E. R. Natural orbital of hydrogen-molecule excited states. J. Chem. Phys. 45, 2560-2576 (1966).

24. Wolniewicz, L. Adiabatic potentials of lowest ${ }^{1} \Pi_{g}$ and ${ }^{1,3} \Delta_{g}$ states of the hydrogen molecule. J. Mol. Spectrosc. 169, 329-340 (1995).

25. Kolos, W. \& Rychlewski, J. Three lowest ${ }^{3} \Pi_{g}$ and three lowest ${ }^{3} \Pi_{u}$ states of the hydrogen molecule. Comput. Methods Sci. Tech. 5, 39-52 (1999).

26. King, J. Jr \& Benson, S. W. Theory of the low-temperature chromatographic separation of the hydrogen isotopes. J. Chem. Phys. 44, 1007-1014 (1966).

27. Kimmel, G. A., Stevenson, K. P., Dohnalek, Z., Smith, R. S. \& Kay, B. D. Control of amorphous solid water morphology using molecular beams. 1 . Experimental results. J. Chem. Phys. 114, 5284-5294 (2001).

28. Kimmel, G. A., Dohnalek, Z., Stevenson, K. P., Smith, R. S. \& Kay, B. D. Control of amorphous solid water morphology using molecular beams. 2. Ballistic deposition simulation. J. Chem. Phys. 114, 5295-5303 (2001).

29. Klaua, M. \& Madey, T. E. The adsorption of $\mathrm{H}_{2} \mathrm{O}$ on clean and oxygen-dosed silver single crystal surfaces. Surf. Sci. 136, L42-L50 (1984).

30. Mokrane, H. et al. Experimental evidence for water formation via ozone hydrogenation on dust grains at 10 K. Astrophys. J. 705, L195-L198 (2009).

\section{Acknowledgements}

We thank T. Kawauchi and T. Okano for assistance with apparatus development, M. Matsumoto and K. Niki for assistance with the experiments, K. Yamakawa for fruitful discussions and M. Wilde for manuscript corrections. This work was supported by Grant-in Aid for Scientific Research (A) of the Japan Society for the Promotion of Science (JSPS). T.S. acknowledges support as a Research Assistant of the Global Centre of Excellence for the Physical Science Frontier of Tokyo University, Japan.

\section{Author contributions}

K.F. planned and organized the project; T.S. designed and developed the experimental system, conducted measurements, analysed the data and devised the theory; T.S. and K.F. discussed the results and wrote the manuscript together.

\section{Additional information}

The authors declare no competing financial interests. Supplementary information accompanies this paper on www.nature.com/naturephysics. Reprints and permissions information is available online at http://npg.nature.com/reprintsandpermissions. Correspondence and requests for materials should be addressed to T.S. or K.F. 